\title{
Erratum
}

\section{Red Dirt Thinking on Child Wellbeing in Indigenous, Rural and Remote Australian Communities: The SpICE Model - ERRATUM}

Kendall Clarke and Marijke Denton

doi: http://dx.doi.org/10.1017/jie.2013.21, Published by Cambridge University Press 13 December 2013

Keywords: Indigenous, specialist, child wellbeing, engagement, rural and remote, collaboration, erratum

The following sentence was published incorrectly in the final version of the article, please see below the corrected sentence:

This ensures that the community: increases its capacity to continue to drive transparent inclusive and partnered servicing; has relevant resources; become informed users of the resources and develop relationships so that there can be continued, remote, support.

\section{Reference}

Kendall Clarke and Marijke Denton (2013). Red Dirt Thinking on Child Wellbeing in Indigenous, Rural and Remote Australian Communities: The SpICE Model "I just don't want my kid to struggle like I did at school". The Australian Journal of Indigenous Education, 42, pp 136-144. doi:10.1017/jie.2013.21. 